\title{
UMA COMPREENSÃO ACERCA DAS POLÍTICAS PÚBLICAS DIRECIONADAS ÀS PESSOAS QUE VIVEM COM O HIV/AIDS NO BRASIL: uma revisão sistemática
}

DOI: 10.22289/2446-922X.V7N1A20

\author{
Marielli Monte Araújo \\ Alexia Jade Machado Sousa ${ }^{1}$ \\ Eduardo Costa Cordeiro \\ Thalia Ariadne Peña Aragão \\ Cláudio Ângelo Ventura \\ Francisco Jander de Sousa Nogueira
}

\section{RESUMO}

O presente trabalho tem como objetivo compreender a lógica da atuação das políticas públicas existentes para pessoas vivendo com HIV/Aids (PVHIV) no Brasil, considerando o percurso histórico da problemática levantada. Pretendemos aqui também focar em uma perspectiva social, considerando as condições de vida e de vulnerabilidade às quais esses sujeitos são submetidos $e$ de que forma as políticas públicas disponíveis respondem às necessidades dessa população. $A$ metodologia utilizada foi à revisão sistemática da literatura que buscou responder como funcionam as políticas públicas para as PVHIV. Diante do exposto é possível compreender que os atravessamentos sobre HIV/Aids são diversos e complexos, evidenciando que não é só uma questão de saúde, mas social, política, econômica e cultural. Portanto, a interprofissionalidade, descentralização do cuidado e abordagem centrada na pessoa são práticas essenciais para a melhoria da qualidade de vida das PVHIV e diminuição da taxa de infecção.

Palavras-chave: Política Pública; HIV/Aids; Sistema Único de Saúde.

\section{AN UNDERSTANDING OF PUBLIC POLICIES AIMED AT PEOPLE LIVING WITH HIV/AIDS IN BRAZIL: a systematic review}

\section{ABSTRACT}

The present work aims to understand the logic of the performance of existing public policies for people living with HIV/AIDS (PLHIV) in Brazil, considering the historical path of the problem raised. We also intend here to focus on a social perspective, considering the conditions of life and vulnerability to which these subjects are submitted and how the available public policies respond to the needs of this population. The methodology used was a systematic literature review that sought to answer how public policies for PLHIV work. Given the above, it is possible to understand that the crossings over HIV/AIDS are diverse and complex, showing that it is not only a matter of health, but social, political, economic and cultural. Therefore, interprofessionality, decentralization of care and

\footnotetext{
${ }^{1}$ Endereço eletrônico de contato: alexiajmachado@gmail.com

Recebido em 19/03/2021. Aprovado pelo conselho editorial para publicação em 11/05/2021.
}

Rev. Psicol Saúde e Debate. Mai., 2021:7(1): 280-292. 
a person-centered approach are essential practices for improving the quality of life of PLHIV and decreasing the rate of infection.

Keywords: Public Policies; HIV/Aids; Health Unic System.

\section{UNA COMPRENSIÓN SOBRE LAS POLÍTICAS PÚBLICAS PARA LAS PERSONAS QUE VIVEN CON VIH/SIDA EN BRASIL: una revisión sistemática}

\section{RESUMEN}

El presente trabajo tiene como objetivo comprender la lógica del desempeño de las políticas públicas existentes para las personas que viven con el VIH/SIDA (PVVIH) en Brasil, considerando la trayectoria histórica del problema planteado. También tenemos la intención de centrarnos aquí en una perspectiva social, considerando las condiciones de vida y vulnerabilidad a las que se someten estos temas y cómo las políticas públicas disponibles responden a las necesidades de esta población. La metodología utilizada fue una revisión sistemática de la literatura que buscaba responder cómo funcionan las políticas públicas para las PVVIH. Dado lo anterior, es posible entender que los cruces sobre el VIH / SIDA son diversos y complejos, lo que demuestra que no es solo una cuestión de salud, sino social, política, económica y cultural. Por lo tanto, la interprofesionalidad, la descentralización de la atención y un enfoque centrado en la persona son prácticas esenciales para mejorar la calidad de vida de las PVVIH y disminuir la tasa de infección.

Palabras clave: Políticas Públicas; VIH/SIDA; Sistema Único de Salud.

\section{INTRODUÇÃO}

A promulgação da Constituição Federal de 1988, a qual traz em seu escopo a saúde como um direito de todos e dever do estado, foi um marco crucial para implantação do Sistema Único de Saúde Brasileiro (SUS) e, consequentemente, para o delineamento de políticas públicas para o enfrentamento ao HIV/Aids (Reis, Araújo, \& Cecílio, 2012). Pautados nos princípios de Universalidade, Integralidade, Equidade e Controle Social, o SUS, em parceria com sociedade civil vem traçando diversas medidas na luta contra a infecção e na melhoria da qualidade de vida de pessoas que vivem com HIV/Aids, desde a distribuição gratuita de medicamentos e a descentralização do cuidado até a criação do Programa Conjunto das Nações Unidas sobre HIV/Aids (UNAIDS) que possui como principal missão liderar, fortalecer e apoiar uma ampla resposta à epidemia de HIV/Aids, a fim de evitar seu avanço (Organização das Nações Unidas, 2020).

Assim como os demais cidadãos, pela Constituição brasileira, as pessoas vivendo com HIV têm obrigações e direitos, entre eles a dignidade humana e o acesso à saúde pública. Em 1989, profissionais da saúde e membros da sociedade civil criaram, com o apoio do Departamento de IST, 
à época era DST, HIV/Aids e Hepatites Virais, a Declaração dos Direitos Fundamentais da Pessoa Portadora do Vírus da Aids. Em 2014, foi publicada a Lei oㅜ 12.984, de 2 de junho de 2014, que define o crime de discriminação aos portadores do vírus da imunodeficiência humana.

Assim, em seu art. $1^{\circ}$ constitui crime as seguintes condutas discriminatórias contra pessoas que vivem com HIV/Aids: recusar, procrastinar, cancelar ou segregar a inscrição ou impedir que permaneça como aluno em creche ou estabelecimento de ensino de qualquer curso ou grau, público ou privado; negar emprego ou trabalho; exonerar ou demitir de seu cargo ou emprego; segregar no ambiente de trabalho ou escolar; divulgar a condição do portador do HIV/Aids ou de doente de aids, com intuito de ofender lhe a dignidade; recusar ou retardar atendimento de saúde (Ministério da Saúde, 2014).

Entretanto, apesar dos avanços no combate ao vírus, o boletim epidemiológico de HIV/Aids de 2019 registrou um aumento crescente na taxa de infecção por HIV/Aids nos últimos anos. De 2017 a 2019 foram registrados 300.429 novos casos de infecção por HIV, destes 207.207 são do sexo masculino e 93.220 do sexo feminino. É importante destacar que a notificação compulsória de infecção por HIV/Aids passa ser obrigatória somente em 2014, podendo mascarar ainda mais o avanço de tal problemática devido à subnotificação e, desse modo, prejudicar o delineamento de políticas públicas eficazes (Presidência da República, 2019).

O crescente aumento nos casos de infecção por HIV é uma questão de saúde pública que engloba diversas variáveis: sociais, políticas, econômicas, entre outras. A síndrome da imunodeficiência humana é transmitida por via sexual, vertical (gestação, parto ou amamentação) e sanguínea (transfusão de sangue, compartilhamento de seringas ou acidentes perfuro cortantes) e é causada por um retrovírus que infecta principalmente os linfócitos T CD4+.

A infecção pelo HIV perpassa diversas fases e depende diretamente da resposta imunológica do indivíduo. Inicialmente, os indivíduos infectados desenvolvem a fase aguda da infecção em que podem surgir sintomas inespecíficos, posteriormente a infecção torna-se assintomática e perdura até o aparecimento da Síndrome da Imunodeficiência Adquirida (Aids) (Martins et al, 2018). O surgimento da infecção por HIV, no Brasil, ocorreu na década de 1980 com o primeiro caso registrado no estado de São Paulo. A princípio negligenciada, a infecção por HIV rapidamente tornou-se um grande problema de saúde pública, não só no Brasil, mas no mundo. Devido à pressão social de ativistas dos diversos movimentos sociais aliado ao processo de disseminação da infecção entre as diversas classes sociais e às vulnerabilizações sociais decorrentes desta, a pandemia causada pelo vírus começou receber a atenção do Estado (Monteiro \& Villela, 2009).

Atualmente, a partir da evolução dos antirretrovirais, os pacientes em terapia (TARV) $(80 \%)$ apresentam supressão viral após um ano de tratamento (Pinto \& Capeletti, 2019). Com isso, houve uma redução da mortalidade e morbidade associadas à infecção e um consequente aumento da 
expectativa de vida das pessoas vivendo com HIV/Aids, assim, a doença migra de estado agudo para condição de doença crônica.

Partindo dessa realidade, o presente trabalho tem como objetivo compreender a lógica da atuação das políticas públicas existentes atualmente para pessoas vivendo com HIV/Aids no Brasil, considerando o percurso histórico da problemática supracitada. Pretendemos aqui também focar em uma perspectiva social, considerando as condições de vida e de vulnerabilidade às quais esses sujeitos são submetidos e de que forma as políticas públicas disponíveis respondem às necessidades dessa população.

\section{MÉTODO}

Trata-se de uma revisão sistemática da literatura que tem como pergunta norteadora: compreender como funcionam as políticas públicas para pessoas vivendo com HIV/Aids no Brasil? Foi escolhida essa metodologia, pois as revisões sistemáticas são úteis particularmente para integrar um conjunto de informações sobre estudos realizados separadamente sobre determinada intervenção, que podem apresentar resultados conflitantes e/ou coincidentes, como também identificar temas que necessitam de evidência, auxiliando na orientação para investigações futuras (Sampaio \& Mancini, 2007).

Estratégia de busca e critério de elegibilidade

Publicações que descreviam sobre as políticas públicas e as pessoas que vivem com HIV/Aids. Foram selecionadas por consenso entre os pesquisadores as estratégias de busca dos artigos nas bases de dados eletrônicas: Literatura Latino-Americana e do Caribe em Ciências da Saúde (Lilacs), Scientific Electronic Library Online (SciELO) e Biblioteca Virtual em Saúde (BVS). Foram utilizados os operadores booleanos "OR" e "AND", que permitiram modular a busca da melhor informação. Os termos encontrados foram aplicados individualmente para refinar e testar a sensibilidade da pesquisa. Assim, foram utilizados os seguintes descritores válidos: (políticas públicas AND pessoas vivendo com HIV/Aids OR assistência).

Para a inclusão dos artigos, foram estabelecidos os seguintes critérios: (1) artigos relacionados às políticas públicas e pessoas vivendo com HIV/Aids; (2) publicados entre 2015 e 2020; (3) estarem disponíveis na forma completa de artigos científicos; e (4) publicados em revistas científicas indexadas nas bases de dados selecionadas; (5) publicados em português. Anais, comunicações breves, monografias, dissertações, teses e boletins foram excluídos.

Foram excluídos também os artigos publicados que não respondiam à pergunta norteadora de acordo com o resumo e/ou o título. Assim, para a extração dos dados necessários a este estudo, 
dos artigos definidos pelos critérios de inclusão, foram analisados os seus resumos. Após análise dos resumos, todos os artigos selecionados foram obtidos na íntegra e posteriormente examinados a fim de que respondessem à pergunta norteadora.

Dos artigos que tinham títulos e resumos que se enquadram na temática e que foram publicados entre 2015 e 2020 destes foram selecionados 21 artigos. Na fase final, os artigos foram lidos na íntegra e foram desconsideradas as duplicatas. Assim, após a aplicação dos filtros dos critérios de inclusão e exclusão, 11 artigos fizeram parte da revisão.

Após estas etapas, foram extraídas as seguintes categorias analíticas: relações entre serviços de saúde e PVHA de grupos populacionais específicos; as respostas ao HIV/Aids a partir da dinâmica social; e a descentralização do cuidado.

\section{RESULTADOS E DISCUSSÃO}

A partir da análise realizada optou-se por produzir um fluxograma que possibilite apresentar todos os artigos escolhidos de maneira sintética para uma visão mais ampla. A Quadro 1 apresenta uma análise geral dos artigos, desde a identificação até a inclusão.

\section{Identificaçào}

Total de artigos encontrados nas bases de dados $(n=27)$

Seleção

Artigos excluídos por duplicidade e por ano $(n=9)$

Elegibilidade

Artigos excluídos após a análise do resumo $(n=4)$

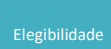

Artigos excluídos avaliados na íntegra $(n=3)$

\section{Inclusão}

Artigos incluídos na revisão sistemática $(n=11)$

Fonte: autores do estudo. 
Quadro 1. Fluxograma do processo de seleção dos artigos.

Após a análise realizada optou-se por produzir um quadro que possibilite apresentar todos os artigos escolhidos para uma visão mais ampla. A Quadro 2 apresenta os artigos escolhidos caracterizando: ano, autores, título e objetivos.

\begin{tabular}{|c|c|c|c|}
\hline ANO & AUTORES & TÍTULO & OBJETIVOS \\
\hline 2015 & $\begin{array}{l}\text { CALAIS \& } \\
\text { PERUCCHI }\end{array}$ & $\begin{array}{l}\text { Aids, infância(s) e políticas } \\
\text { públicas: a construção de } \\
\text { sujeitos através de discurso }\end{array}$ & $\begin{array}{l}\text { Investigar os discursos que constituem as } \\
\text { políticas públicas em saúde no Brasil } \\
\text { voltadas à questão da Aids na infância e } \\
\text { seus dispositivos de saber poder que } \\
\text { constroem posições de sujeito }\end{array}$ \\
\hline 2015 & SCHOR & $\begin{array}{l}\text { As primeiras respostas à } \\
\text { epidemia de aids no Brasil: } \\
\text { influências dos conceitos de } \\
\text { gênero, masculinidade dos } \\
\text { movimentos sociais. }\end{array}$ & $\begin{array}{l}\text { Apresentar e discutir as concepções de } \\
\text { gênero e masculinidade, e como estas foram } \\
\text { fundamentais para a formulação de uma } \\
\text { identidade e de movimentos que marcaram } \\
\text { as primeiras respostas do Estado brasileiro à } \\
\text { epidemia da aids. }\end{array}$ \\
\hline 2016 & $\begin{array}{l}\text { ZAMBENEDETTI } \\
\text { \& SILVA }\end{array}$ & $\begin{array}{l}\text { Descentralização da } \\
\text { atenção em HIV/Aids para a } \\
\text { atenção básica: tensões e } \\
\text { potencialidades }\end{array}$ & $\begin{array}{c}\text { Identificar e discutir as tensões que } \\
\text { permeiam o processo de descentralização do } \\
\text { diagnóstico para HIV e acompanhamento das } \\
\text { pessoas vivendo com HIV/Aids para a } \\
\text { atenção básica. Foi realizada uma pesquisa } \\
\text { de base qualitativa, sob o viés analítico } \\
\text { institucional, em Porto Alegre-RS. }\end{array}$ \\
\hline 2016 & $\begin{array}{l}\text { ALENCAR \& } \\
\text { CIOSAK }\end{array}$ & $\begin{array}{l}\text { Aids em idosos: motivos que } \\
\text { levam ao diagnóstico tardio. }\end{array}$ & $\begin{array}{l}\text { Investigar entre os idosos vivendo com } \\
\text { HIV/Aids e os profissionais de saúde, quais } \\
\text { são os motivos que levam ao diagnóstico } \\
\text { tardio da infecção pelo HIV nos idosos. }\end{array}$ \\
\hline 2017 & $\begin{array}{l}\text { SILVA \& } \\
\text { BARBOSA }\end{array}$ & $\begin{array}{l}\text { Diferenças e similaridades } \\
\text { entre mulheres que vivem } \\
\text { com HIV: aportes do estudo } \\
\text { GENIIH para a atenção à } \\
\text { saúde sexual e reprodutiva. }\end{array}$ & $\begin{array}{c}\text { A identificação de contextos de } \\
\text { vulnerabilidade e a integração de serviços de } \\
\text { testagem anti-HIV e de saúde sexual e } \\
\text { reprodutiva devem compor as linhas de } \\
\text { cuidado às mulheres, tanto nos serviços } \\
\text { especializados quanto nos de atenção } \\
\text { básica. }\end{array}$ \\
\hline 2018 & $\begin{array}{l}\text { MELO, MAKSUD } \\
\text { \& AGOSTINI }\end{array}$ & $\begin{array}{l}\text { Cuidado, HIV/Aids e } \\
\text { atenção primária no Brasil: } \\
\text { desafio para a atenção no } \\
\text { Sistema Único de Saúde? }\end{array}$ & $\begin{array}{l}\text { Contextualizar o recente processo de } \\
\text { descentralização do cuidado às pessoas com } \\
\text { HIV/Aids para o âmbito da APS no Brasil - } \\
\text { tema ainda escasso na literatura científica - e } \\
\text { problematizar potencialidades e desafios } \\
\text { como o direito das pessoas com HIV/Aids. }\end{array}$ \\
\hline 2018 & $\begin{array}{l}\text { TEIXEIRA, } \\
\text { PAULINO, } \\
\text { RAIMONDI, } \\
\text { CROVADO \& } \\
\text { PRADO }\end{array}$ & $\begin{array}{c}\text { Entre o segredo e as } \\
\text { possibilidades do cuidado: } \\
\text { (re)pensando os silêncios } \\
\text { em torno das narrativas das } \\
\text { travestis sobre HIV/Aids }\end{array}$ & $\begin{array}{c}\text { Discutir os sentidos sobre a aids que } \\
\text { circulam, tanto entre as travestis como nos } \\
\text { aparatos da política pública, impactando na } \\
\text { adesão delas às ofertas oficiais de cuidado } \\
\text { em saúde. }\end{array}$ \\
\hline 2018 & LUI \& LEAL & $\begin{array}{l}\text { Instituições participativas e } \\
\text { seus efeitos nas políticas } \\
\text { públicas: estudo do Comitê } \\
\text { de Mortalidade por aids de } \\
\text { Porto Alegre }\end{array}$ & $\begin{array}{l}\text { Conhecer a percepção dos membros do } \\
\text { Comitê Municipal de Mortalidade por aids em } \\
\text { Porto Alegre sobre como essa instituição } \\
\text { participativa tem consequências na melhoria } \\
\text { do atendimento às pessoas que vivem com } \\
\text { HIV/Aids. }\end{array}$ \\
\hline 2018 & $\begin{array}{l}\text { MAGNABOSCO, } \\
\text { LOPES, } \\
\text { ANDRADE, } \\
\text { BRUNELLO, }\end{array}$ & $\begin{array}{l}\text { Assistência ao HIV/Aids: } \\
\text { análise da integração de } \\
\text { ações e serviços de saúde }\end{array}$ & $\begin{array}{l}\text { Analisar a integração das ações e serviços } \\
\text { de saúde na assistência às Pessoas Vivendo } \\
\text { com HIV/Aids (PVHA), pelos Serviços de }\end{array}$ \\
\hline
\end{tabular}




\begin{tabular}{|c|c|c|c|}
\hline & $\begin{array}{l}\text { MONROE \& } \\
\text { VILLA }\end{array}$ & & $\begin{array}{c}\text { Atenção Especializada ao HIV/Aids (SAE) de } \\
\text { Ribeirão Preto-SP. }\end{array}$ \\
\hline 2019 & $\begin{array}{l}\text { MENEZES, } \\
\text { POMPILIO \& } \\
\text { ANDRADE }\end{array}$ & $\begin{array}{l}\text { A integração do cuidado: } \\
\text { dificuldades e perspectivas }\end{array}$ & $\begin{array}{c}\text { Caracterizar a integração do cuidado entre as } \\
\text { equipes de Assistência Domiciliar } \\
\text { Terapêutica em HIV/Aids e a Estratégia } \\
\text { Saúde da Família na perspectiva de } \\
\text { profissionais, pacientes e cuidadores. }\end{array}$ \\
\hline 2019 & $\begin{array}{l}\text { PINTO \& } \\
\text { CAPELETTI }\end{array}$ & $\begin{array}{l}\text { Reorganização do modelo } \\
\text { de atenção às pessoas } \\
\text { vivendo com HIV: a } \\
\text { experiência do município de } \\
\text { Florianópolis/SC }\end{array}$ & $\begin{array}{c}\text { Melhorar os indicadores de controle da } \\
\text { doença, destacando-se fatores locais como a } \\
\text { rede de atenção primária estruturada, a } \\
\text { gestão eficiente e a busca da qualificação } \\
\text { profissional. }\end{array}$ \\
\hline
\end{tabular}

Fonte: Autores do estudo.

Quadro 2. Caracterização dos estudos.

Todos os artigos trazem questões persistentes e atuais que precisam ser revisitados com atenção para uma atenção primária mais próxima das realidades com os sujeitos, acrescentando suas necessidades.

Os trabalhos selecionados trazem muitos pontos a serem debatidos, alguns artigos são singulares ao trazer os atravessamentos das políticas públicas em grupos bem específicos como crianças, idosos, mulheres, travestis e transexuais, questão que será descrita em um dos tópicos da discussão. No entanto, eles também corroboram sobre algumas questões relacionadas às políticas públicas, revelando os pontos de tensão que perduram durante os anos e que dificultam os processos para uma atenção à saúde mais ampla e conectada com as realidades das PVHA. Nessa perspectiva, na tentativa de extrair o máximo das temáticas apresentadas nos artigos, foram construídos eixos de discussão onde é pretendido tencionar os nós apresentados pelos estudos.

Relações entre serviços de saúde e PVHA de grupos populacionais específicos

Os estudos realizados por Perucchi e Calais (2015) e Alencar e Cosak (2016), que abordam crianças e idosos vivendo com HIV/Aids, respectivamente, trazem discussões acerca dos principais desafios enfrentados por esses grupos. Segundo os mesmos, estigmas e estereótipos criados em torno desses grupos afetam diretamente o diagnóstico, o tratamento e a qualidade de vida (QV) das pessoas que vivem com o HIV/Aids. Esse processo de estigmatização acarreta um reducionismo do ser ao âmbito puramente biologista e se difere dos objetivos preconizados pela Política Nacional de Humanização $(\mathrm{PNH})$ que busca contemplar um cuidado holístico e pautado pelos princípios de integralidade e equidade do SUS (Rocha et al, 2015).

Para Perucchi e Calais (2015), o paradoxo de visibilidade e invisibilidade, presente no acompanhamento de crianças vivendo com HIV/Aids, revela um dos pontos de crítica às políticas públicas. De forma que suas ações, segundo os autores, estão direcionadas prioritariamente à 
doença, ao estado de saúde dos sujeitos, descartando as demais atribuições e singularidades. Essa forma de relação contribui para as ações dos profissionais de saúde se restringir somente a questão biológica. Nesse meio, a hierarquia de saber e poder se fazem presentes e constroem os atos das políticas públicas em um campo permeado de outras demandas que são facilmente esquecidas. Ademais, essa é uma realidade duramente criticada no estudo diante de um trabalho que deve seguir de forma estratégica e elaborada, construindo vínculos com as pessoas que vivem com HIV/Aids (PVHA).

No que diz respeito aos idosos vivendo com HIV/Aids, o cenário é ainda mais preocupante. De acordo com os autores Alencar e Cosak (2016), a sexualidade dos idosos é vista como um grande tabu, a detecção da doença é prevalente nos níveis secundário e terciário da atenção à saúde, tal fato demonstra as dificuldades que a Atenção Primária à Saúde (APS) enfrenta para realizar o diagnóstico precoce neste grupo. Os mesmos autores esclarecem que um dos obstáculos consiste na diferença de idade e de gênero, pois profissionais do sexo feminino revelaram não se sentir à vontade para falar de tal conteúdo com os idosos do sexo masculino, tocando no assunto somente em tempos oportunos, por exemplo, em épocas de campanhas como o "Fique Sabendo", proporcionada pelo Ministério da Saúde (MS).

É preciso ressaltar que existem outros fatores que contribuem no fortalecimento dessa relação entre pessoas de maior idade e profissionais da saúde, como a crença de que os idosos não possuem uma vida sexual ou que são assexuados. Embora a sexualidade e a vida afetiva tenham um impacto crucial na qualidade de vida das PVHA, sendo seu conhecimento amplo indispensável para o delineamento de estratégias pelos profissionais que melhorem a qualidade de vida dos usuários (Beltrão et al, 2020). É então, com esse cenário do cotidiano, cercado de mitos e preconceitos, que é possível entender as barreiras que contribuem para a efetividade das políticas públicas para a promoção e prevenção em saúde (Alencar \& Cosak, 2016).

No tocante ao recorte de gênero, as mulheres idosas são mais suscetíveis a infecções sexualmente transmissíveis (IST) quando comparadas aos homens, especialmente se tiverem sido expostas a ISTs anteriormente (Andrade et al, 2017). Existe uma urgente demanda para a constante qualificação dos recursos humanos da saúde, mais especificamente da APS. Isso se justifica por ser a APS o espaço privilegiado para a promoção, prevenção e diagnóstico precoces, já que suas relações com as mulheres em vulnerabilidade são mais próximas do território que os demais níveis de atenção (Pinho, Cabral, \& Barbosa, 2017).

Junto a esta questão, Cerqueira-Santos et al. (2010), Nogueira e Aragão (2019) trazem à tona o fato de que travestis e transexuais enfrentam barreiras no acesso às instituições de saúde, que vão desde a estigmatização e a insistência em associar a prostituição como um atributo dessa população e consequentemente colocá-las como grupo de maior risco para infecção pelo HIV/Aids; até a recusa em reconhecer suas identidades, deslegitimando-as. De acordo com os estudos 
supracitados, o relato desses sujeitos claramente descrevia discriminações nos serviços de saúde, onde o resultado mais comum era o abandono ao tratamento resistindo fortemente ao retorno.

Posto isto, reconhece-se a urgência por uma atenção à saúde ampla que seja capaz de integrar o cuidado, deslocando da centralidade da atenção a doença e centralizando o usuário, bem como seus desejos, aspirações, modos de vida e contingências individuais. Tais questões esbarram ainda nas relações construídas socialmente e que estão cercadas por estereótipos, estigmas, mitos e preconceitos, como já foi citado no início deste tópico, e que reflete fortemente nas relações sociais e atuação profissional junto a PVHA. Por isso, merecem serem revistas e repensadas de forma a criar uma atenção mais próxima do que se visualiza com a PNH. Sendo assim, diante dessa realidade, as variáveis que contribuem para a mortalidade por HIV/Aids são múltiplas e nunca devem ser considerados isoladamente, mas através da intrínseca e complexa relação entre os fatores sociais, culturais, econômicos, de gênero e sexualidade (Leal \& Luí, 2018). Portanto, a melhor articulação dessas relações é indispensável para que as instituições de cuidado estejam preparadas para as barreiras que existem no percurso que vai do diagnóstico até o tratamento.

As respostas ao HIV/Aids a partir da dinâmica social

Em meio ao processo de redemocratização e Reforma Sanitária, as lutas e reivindicações da sociedade foram decisivas para criação de políticas públicas e a construção do Sistema Único de Saúde (SUS) (Rolim et al, 2013). A participação social é um dos princípios do SUS que compreende a sociedade como agente ativo na construção desse sistema. No entanto, Dantas (2018) aponta que apesar do caráter crucial da participação e do controle social na criação do SUS e das estratégias de enfrentamento a epidemia de HIV/Aids, as reivindicações feitas ficaram aquém do esperado, não contemplando os interesses da classe trabalhadora.

Santos e Schor (2015), defende que a resposta ao HIV/Aids não é igual em todas as sociedades e por isso são traçadas a partir das relações já existentes, ou seja, a doença é construída pela sociedade. Por essa razão, tanto a maneira de perceber a doença como as resoluções para ela são singulares de cada cultura. A sociedade brasileira é estruturada sob a ótica de uma hegemonia heterossexual patriarcal e machista, desse modo, os recortes de gênero, raça, sexualidade e classe são essenciais para o entendimento da resposta estatal frente a epidemia dos anos 80 .

Ainda, segundo o mesmo autor, a discussão sobre os papéis de gênero e sexualidade começou a ganhar um destaque importante antes da epidemia com os movimentos que apareceram a partir das lutas de grupos homossexuais. Entretanto, o surgimento do vírus associado ao estigma, aumentou o preconceito em torno desses grupos. Apesar disso, a democratização da infecção pelo HIV/Aids, antes restrita a grupos minoritários, mobilizou lutas para a garantia de direitos às pessoas 
vivendo com HIV/Aids. Foi então, partindo da ordem das tensões dentro da própria sociedade que foi possível obterem respostas a este problema. Dessa forma, foram surgindo novos modelos de se pensar as políticas públicas e a participação da sociedade civil se tornou um diferencial para o enfrentamento da epidemia.

Nesse contexto das produções de respostas ao HIV/Aids, o trabalho de Leal e Luí (2018) debate o quanto a participação social é importante tanto para a criação de políticas públicas como para o combate, ou seja, se elabora tanto no campo dos pedidos e reivindicações como no de luta. Destacam, portanto, o controle social como estratégico e organizativo para gestão pública e elaboração de políticas de enfrentamento. Reafirmam também, que as lutas sociais trazem resultados, e quanto mais tempo de duração as instituições de bases tem, como os Comitês, mais elas ganham força e voz.

\section{A Descentralização do Cuidado}

A Estratégia Saúde da Família (ESF) foi criada 1994, mais de uma década após a epidemia de HIV/Aids. De acordo com o estudo de Melo, Maksud e Agostini (2018), o modelo de atenção a PVHIV, nos anos 80, acontecia em serviços específicos, sendo então centralizado, pautado no manejo ambulatorial. Ainda alegam que essa estratégia que se apresenta de forma pontual e centralizada, que dá privilégio à infecção, pautada pelo modelo biomédico e que refletiu negativamente no combate ao vírus, resultou na centralização da doença. Cuidar da infecção é importante, porém, como apontam os demais estudos, outros fatores se fazem presentes e demandam ser abordados.

Segundo Menezes et al. (2018), a ação de descentralização foi percebida como necessária e importante na construção de diálogos acontecendo entre diferentes setores como órgão governamentais, gestores, profissionais e PVHA, indicando uma cadeia de cuidado que exige da gestão e dos serviços. Acrescentam que os gestores são importantes estimuladores e apoiadores para a inclusão e a efetivação da descentralização do cuidado com PVHA, sendo a Atenção Primária à Saúde (APS) a base para a integração com os demais níveis de atenção e criação de linhas de cuidados mais próximas das demandas existentes.

Os debates traçados no estudo de Zambenedetti e Silva (2016) corroboram com o ato de descentralização, se revelando com diferentes faces e como um fenômeno complexo que envolve uma mobilização subjetiva do cuidador, desenvolvendo uma dinâmica de corresponsabilização pelo cuidado, e não somente uma transferência de responsabilidade.

Não obstante, a descentralização impõe algumas dificuldades e barreiras que potencializam algumas tensões. De acordo com Magnabosco et. al. (2018), esses percalços podem ser evidenciados pela existência de equipes incompletas, as quais se alternam frequentemente, além 
da sobrecarga no trabalho. Desse modo, convém admitir que a mudança constante de equipes de referência interfere diretamente no vínculo com os usuários. Outro ponto são as lacunas na rede que dificultam a compreensão das demandas dos usuários e, consequentemente, a resolutividade das mesmas. Por outro lado, as potencialidades necessitam ser realçadas. A referência e contrarreferência são apresentadas como mecanismos de fortalecimento da rede assim como o matriciamento - atribuições de grande potência especialmente quando realizadas por equipes pautadas no trabalho interprofissional -, destacado como diferencial na resolutividade, ajuda das demandas profissionais e para distribuição dos papéis nesse cenário (Magnabosco et al, 2018).

Diante disso, Melo, Maksud e Agostini (2018) corroboram com a ideia de que a APS é a porta de entrada do SUS e por isso tem a missão de coordenar, manter e ampliar ações de prevenção, promoção, cura, manutenção da saúde, reabilitação e redução de danos e são as Equipes de Saúde da Família que fazem a organização desse meio. Entretanto, afirmam que as disparidades socioeconômicas e regionais constituem importantes barreiras para a operacionalização dos serviços, mostrando a complexidade do trabalho, além elucidar que diversas variáveis estão implicadas no processo de construção e ampliação da atenção às PVHA (Melo, Maksud, \& Agostini, 2018).

Dessa maneira, para além de uma transformação da atenção, como a descentralização é necessária uma transformação em ato, os movimentos sociais já foram apontados e percebidos como protagonistas. Contar com a participação social é o ponto de partida.

\section{CONSIDERAÇÕES FINAIS}

Compreende-se que os trabalhos aqui expostos trazem questões ainda pertinentes e com potencial de discussão e avaliação nas políticas públicas. Evidenciou-se que são diversos e complexos os atravessamentos sobre a HIV/Aids, aqui tratados em grupos específicos, o HIV/AIDS tem as suas nuances diversas ao acometer idosos, mulheres, travestis, transexuais, etc. Os estudos aqui utilizados reforçam ainda que essa é uma questão não só de saúde, mas atravessada social, política, econômica e culturalmente.

Desse modo, é importante uma reflexão sobre as práticas dentro das políticas públicas - e na atuação - para os profissionais que acolhem essa população vivendo com HIV/Aids. Nesse sentido, a discussão em torno da Interprofissionalidade é campo comum no tocante à atenção e cuidado em saúde. As experiências interprofissionais se mostraram exitosas, pois propõem a integralidade a partir da atuação conjunta de diferentes núcleos de saberes.

Isso se justifica pela imperatividade da descentralização da abordagem biológica, a qual até os dias atuais se prevalece em conhecimentos e práticas (Monteiro et al., 2019). Além do mais, a 
problemática do HIV/Aids é multifatorial, exigindo uma abordagem também multifatorial, proporcionada comumente por experiências interprofissionais.

No contexto atual, o que se propõe enquanto prática considerando as características relacionadas à prevenção e tratamento do HIV/Aids é o que Parker (2019) chama de Pedagogia da Prevenção, pautada nos princípios de solidariedade, dos direitos humanos e na aproximação entre saberes científicos e populares.

\section{REFERÊNCIAS}

Alencar, R. A., \& Ciosak, S. I. (2016). Aids em idosos: motivos que levam ao diagnóstico tardio. Revista Brasileira de Enfermagem, 69(6), 1140-1146.

Andrade, J., Ayres, J. A., Alencar, R. A., Duarte, M. T. C., \& Parada, C. M. G. D. L. (2017). Vulnerabilidade de idosos a infecções sexualmente transmissíveis. Acta Paulista de Enfermagem, 30(1), 8-15.

Amarante, P. (1996). O homem e a serpente: outras histórias para a loucura e a psiquiatria. SciELOEditora FIOCRUZ.

Beltrão, R. P. L., da Silva, A. C. B., Nogueira, F. J. S., \& Mouta, A. A. N. (2020). Saúde e qualidade de vida das pessoas vivendo com HIV/aids: uma revisão narrativa dos últimos 15 anos. Revista Eletrônica Acervo Saúde, (40), e2942-e2942.

Cerqueira-Santos, E., Calvetti, P. U., Rocha, K. B., Moura, A., Barbosa, L. H., \& Hermel, J. (2010). Percepção de usuários gays, lésbicas, bissexuais e transgêneros, transexuais e travestis do Sistema Único de Saúde. Interamerican Journal of Psychology, 44(2), 235-245.

Dantas, A. V. (2018). Saúde, luta de classes e o 'fantasma'da Reforma Sanitária Brasileira: apontamentos para sua história e crítica. Saúde em Debate, 42, 145-157.

de Calais, L. B., \& Perucchi, J. (2015). Aids, Infância (s) e Políticas Públicas: A Construção de Sujeitos Através do Discurso. Psicologia em Estudo, 20(2), 165-176.

Leal, A. F., \& Lui, L. (2018). Instituições participativas e seus efeitos nas políticas públicas: estudo do Comitê de Mortalidade por Aids de Porto Alegre. Saúde e Sociedade, 27, 94-105.

Martins, V., Batista, R. A., Sehnem, G. D., Merigo, G. K., Rezer, J. F. P., \& da Silva, V. A. M. (2018). Infecções sexualmente transmissíveis (IST): estratégias de intervenção para promoção de saúde. Anais do Salão Internacional de Ensino, Pesquisa e Extensão, 10(2).

Magnabosco, G. T., Lopes, L. M., Andrade, R. L. D. P., Brunello, M. E. F., Monroe, A. A., \& Villa, T. C. S. (2018). Assistência ao HIV/aids: análise da integração de ações e serviços de saúde. Escola Anna Nery, 22(4).

Melo, E. A., Maksud, I., \& Agostini, R. (2018). Cuidado, HIV/Aids e atenção primária no Brasil: desafio para a atenção no Sistema Único de Saúde?. Revista Panamericana de Salud Pública, 42, e151.

Menezes, K. M. D., Pompilio, M. A., \& Andrade, S. M. O. D. (2019). A integração do cuidado: dificuldades e perspectivas. Rev. enferm. UFPE on line, 1052-1063. 
Ministério da Saúde (2019). Boletim Epidemiológico HIV/Aids. Brasília, DF: Ministério da Saúde.

Monteiro, A. L., \& Villela, W. V. (2009). A criação do Programa Nacional de DST e Aids como marco para a inclusão da idéia de direitos cidadãos na agenda governamental brasileira. Revista Psicologia Política, 9(17), 25-45.

Monteiro, S. S., Brigeiro, M., Vilella, W. V., Mora, C., \& Parker, R. (2019). Desafios do tratamento como prevenção do HIV no Brasil: uma análise a partir da literatura sobre testagem. Ciência \& Saúde Coletiva, 24, 1793-1807.

Nogueira, F. J. S., \& Aragão, T. A. P. (2019). Política Nacional de Saúde Integral LGBT: o que ocorre na prática sob o prisma de usuários (as) e profissionais de saúde. Saúde e Pesquisa, 12(3), 463-470.

Organização das Nações Unidas. UNAIDS Brasil, 2020. Página Inicial. Disponível em:< https://unaids.org.br/>. Acesso em: 24 de maio de 2020.

Parker, R. (2019). Estigmas do HIV/Aids: novas identidades e tratamentos em permanentes sistemas de exclusão. Revista Eletrônica de Comunicação, Informação e Inovação em Saúde, 13(3).

Pinho, A. D. A., Cabral, C. D. S., \& Barbosa, R. M. (2017). Diferenças e similaridades entre mulheres que vivem e não vivem com HIV: aportes do estudo GENIH para a atenção à saúde sexual e reprodutiva. Cadernos de Saúde Pública, 33, e00057916.

Pinto, V. M., \& de Mattos Capeletti, N. (2019). Reorganização do modelo de atenção às pessoas vivendo com HIV: A experiência do município de Florianópolis/SC. Revista Brasileira de Medicina de Família e Comunidade, 14(41), 1710-1710.

Presidência da República (2014). Lei n. 12.984/2014. Brasília, DF: Presidência da República. Disponível em: <http://www.planalto.gov.br> Acesso em: 23 abr. 2020.

Reis, D. O., Araújo, E. C. D., \& Cecílio, L. C. D. O. (2012). Políticas Públicas de Saúde no Brasil: SUS e pactos pela Saúde. Módulo Político Gestor. Disponível em: http://www. unasus. unifesp. br/biblioteca_virtual/esf/1/modulo_politico_gestor/Uni dade_4. pdf. Acesso em, 21 junho 2020.

Rocha, G. S. D. A., Angelim, R. C. D. M., Andrade, Â. R. L. D., Aquino, J. M., Abrão, F. M. D. S., \& Costa, A. M. (2015). Cuidados de enfermagem aos indivíduos soropositivos: reflexão à luz da fenomenologia. Revista Mineira de Enfermagem, 19(2), 258-265.

Rolim, L. B., Cruz, R. D. S. B. L. C., \& Sampaio, K. J. A. D. J. (2013). Participação popular e o controle social como diretriz do SUS: uma revisão narrativa. Saúde em debate, 37(96), 139 147.

Sampaio, R. F., \& Mancini, M. C. (2007). Estudos de revisão sistemática: um guia para síntese criteriosa da evidência científica. Brazilian Journal of Physical Therapy, 11(1), 83-89.

Santos, R. C. S., \& Schor, N. (2015). As primeiras respostas à epidemia de aids no Brasil: influências dos conceitos de gênero, masculinidade e dos movimentos sociais. Psicologia Revista, 24(1), 45-59.

Zambenedetti, G., \& Silva, R. A. N. D. (2016). Descentralização da atenção em HIV-Aids para a atenção básica: tensões e potencialidades. Physis: Revista de Saúde Coletiva, 26, 785-806.

Rev. Psicol Saúde e Debate. Mai., 2021:7(1): 280-292. 